Review

\title{
The good among the bad and the ugly: the unsung heroes in ER stress, GPCR and EMT
}

\author{
Rohit Gundamaraju*, Wenying Lu and Rajaraman Eri \\ 1. School of Health Sciences, University of Tasmania, Launceston, Tasmania, Australia 7248. \\ * Correspondence:rohit.gundamaraju@utas.edu.au
}

\begin{abstract}
The majority of cellular responses to external stimuli are mediated by receptors such as $G$ protein-coupled receptors (GPCRs) and systems including endoplasmic reticular stress (ER stress). Since GPCR signalling is pivotal in numerous malignant pathologies, they are targeted by a number of clinically used drugs. Cancer cells often negatively modulate GPCRs in order to survive, proliferate and to disseminate. Similarly, numerous branches of the unfolded protein response (UPR) act as pro-survival mediators and are involved in promoting cancer progression via mechanisms such as epithelial mesenchymal transition (EMT). However, there are a few proteins among these groups which impede deleterious effects by orchestrating the pro-apoptotic phenomenon and paving a therapeutic pathway. The present review exposes and discusses such critical mechanisms and some of the key processes involved in carcinogenesis.
\end{abstract}

Keywords: ER stress; GPCR; EMT; cancer progression; migration; cancer

\section{Introduction}

G protein-coupled receptors (GPCR) contribute to a number of physiological capabilities during tumorigenesis [1] and are vastly involved in the control of virtually all cell types. Their structure allows for binding of highly diverse ligands, thus they are considered to be the most druggable family of proteins. Loss of balance in the activation of these receptors may result in triggering of conditions such as carcinogenesis. Mechanisms such as GTP hydrolysis, second messenger related protein kinases (e.g. PKA and PKC), G-protein-coupled receptor kinases (GRKs), and arresting prevent the malfunctioning of GPCR signalling. GRKs in general phosphorylate their target GPCR in order to prevent excessive cellular signalling. GRKs are considered to be negative regulators of GPCR activity and are involved in tumorigenesis through processes such a cell death, proliferation, invasion and vascularisation [2]. Nearly 108 GPCR targets are available but fewer than eight are in the anti-cancer class[3]. Moreover, understanding how GRKs regulate GPCR activity may greatly aid in understanding oncogenesis and respected therapeutics.

Activation of GPCR expressed in various cells has been found to stimulate ER stress [4]. Arrestin1(ARR-1), the GPCR protein in Caenorhabditis elegans, was studied in order to investigate homeostasis. It was noted that ARR-1 was essential for GPCR signalling which controls unfolded protein response (UPR) and various neural pathways concerning ER stress were governed by GPCR. NPR-1, in a neural circuit setting, controls the p38/PMK-1 MAPK pathway required for innate immunity[5]. Similarly, OCTR-1 also regulates the p38/PMK-1 MAPK pathway and other UPR pathways [6]. GPCRs were reported to be key players not only in cancers but also in inflammationrelated diseases such as ulcerative colitis and Crohn's disease, and OGR1 was identified as a classic example of GPCR protein expressed in gut-related inflammatory diseases. Surprisingly, OGR1 aided in the regulation of ER stress through the IRE1 $\alpha$-JNK signalling pathway and blockage of autophagosomal degradation [4]. This body of evidence forms a strong understanding between GPCR and ER stress. 
The course of linkage of GPCR and ER stress to EMT progression is of interest in cancer therapeutics (Figure1). Furthermore, in cancer progression, chemotactic migration is regarded as a key aspect, and it has been found that the activation of hemotactic GPCRs led to impaired autophagosome biogenesis in U87 cells which aided in cell migration [7].

In addition, epithelial-mesenchymal transition (EMT) also plays an important role in the development of resistance to EGFR tyrosine kinase inhibitors (TKIs), such as gefitinib, in NSCLC [8]. Hence, the functional crosstalk between GPCRs and EGFR linked to EMT could be a potential target for inhibiting EMT-associated metastasis in lung cancer. We have previously shown that EMT is an active process in NSCLC and mutually associated with airway disease [9]. EGFR and EMT-related protein expressions were markedly high in the peripheral leading edge of NSCLCs and related to tumour characteristics associated with poor prognosis. The relationships between EMT-related tumour biomarker expression and those in the airway epithelium and Rbm provide a background for utility of airway changes in clinical settings. The functional crosstalk between GPCRs and EGFR also contributes to the progression of other cancers, such as colon, breast, and head and neck tumours $[10,11]$. Another GPCR super family member, GPR171, identified as a potential tumour-promoting gene, is also over expressed in lung cancer[12]. Studies have shown that GPR171 enhanced proliferation[12] and metastasis of lung cancer[12,13] in an EGFR-independent manner. Therefore, it is also proposed that combined inhibition of GPR171 and EGFR might be a promising strategy for lung cancer treatment. GPCRs are also known to be involved in tumour progression by coupling with the Gs-, G $\alpha$ - and Gq- protein-signalling pathways. For instance, GPCRs can interact with Rho GTPase by coupling with the Gaq signalling pathway which is involved in cell migration and invasion[14][15]. GPR78 is an orphan GPCR and a Gs-coupled receptor in regulating the cellular level of cAMP[16] and it has been found to be expressed in several lung cancer cell lines[17]. The mRNA level of GPR78 was also found to be increased in lung cancer cells[17]. The over expression of GPR78 in lung cancer cells promoted cell migration by inducing the activation of RhoA and Rac1, which are small signallingG proteins of Rho GTPase[18] through the Gaq-signalling pathway[17]. GPR78 knockouts significantly suppressed the cell migration in metastatic lung cancer cell lines[17]. It has been understood that the activation of RhoA and Rac1 signalling was likewise associated with the mTORC2 and mTORC2 activity in regulating EMT in the metastasis of colorectal cancer cells[19]. GPCRs knockouts could also potentially suppress EMT-associated metastasis in lung cancer via the Rho GTPase pathway, however further work is needed in this direction.

On the other hand, the orchestrating role of ER stress in EMT initiation has been well established. ER stress induces EMT in AECs, at least in part through Src-dependent pathways, thereby demonstrating its role in fibroblast accumulation in pulmonary fibrosis [20]. Hypoxia was another factor driving pro-EMT transcription factors, as well as the activation of ER stress markers both in vivo in rat lungs and in vitro in aleveolar epithelial cells [21]. Hence hypoxia and intracellular calcium are both involved in EMT induction of AECs, mainly through the activation of ER stress and hypoxiainduced factor (HIF)-signalling pathways. Enhanced ER stress was responsible for induction of EMT in human lens epithelial cells [22]. Histone deacetylases (HDACs) participate in the regulation of dynamic equilibrium state of histone or non-histone acetylation/deacetylation and their deacetylase activity could be inhibited by histone deacetylase inhibitor (HDACi). ER stress-inducing agents such as tunicamycin and bleomycin induced ER stress and EMT in lung epithelial cells via the upregulation of HDACs which was proved by employing the HDAC inhibitor via the Smad pathway [23]. ER stress employed its arms in the process of EMT induction. The Src-dependent pathway was previously shown in pulmonary fibrosis [20]. The inositol-requiring protein 1 (IRE1)-XBP1 pathway was found to promote EMT via Snail expression in pulmonary fibrosis. The interrelationship between GPCRs, ER stress and EMT is nearer to a triangle. Therefore, in this review, we intend to revisit some of the key candidates in the pathways such as GPCR, ER stress and EMT which have been developed in order to modulate and have been tested in disease models. 


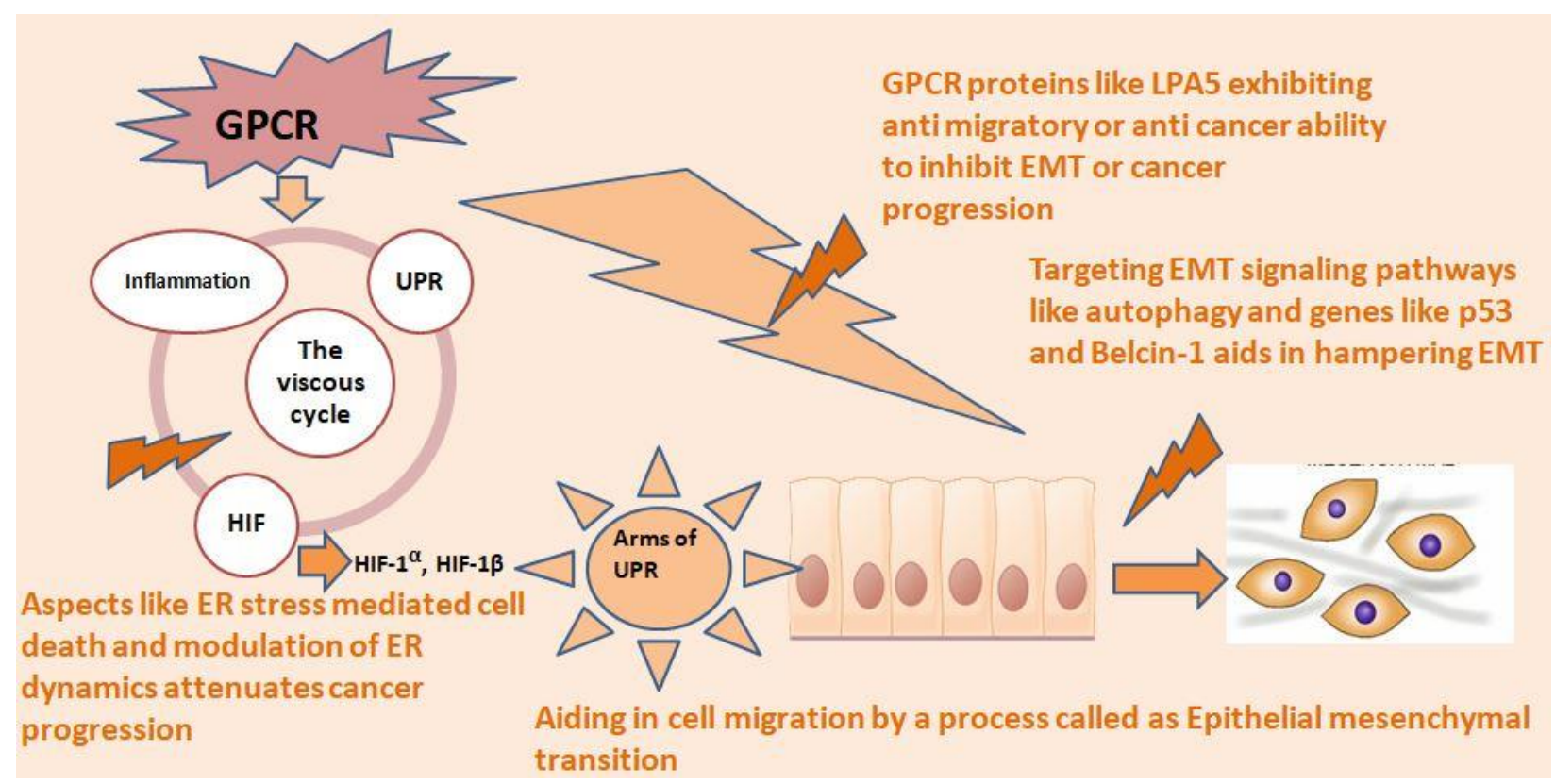

Figure 1. the interrelation between GPCR, ER stress and EMT.

\section{LPA5 is a friend in need among GPCRs}

The long-term expression between LPA5 and cancer progression has been the subject of debate. LPA treatment was found to reduce cell survival, this having been proved via LPA5 knockdown. A secondary messenger, cAMP, has also been implicated widely in cell-death decisions, acting as a switch, and LPA5 facilitates CAMP accumulation, thereby reducing cell survival. LPA5-mediated signalling also reduced cell survival in MG-63 cells. LPA5 combines with Gq and G12/13 which in turn activates Rho-mediated signalling emerging in lessened survival in MG-63 cells [24].

LPA vastly dictates embryonic development which is an indirect effector of tumour growth, angiogenesis and metastasis, and a serendipitous finding showed cell migration being impeded by LPA in B16F10 melanoma cells. Interestingly, LPA5 mediated the inhibitory effect via rise in cAMP and co-activation of protein kinase A (PKA). LPA5 was importantly considered to be an antimigratory agent due to its ability to elevate cAMP in both original and transfected cells [25]. Similarly, pancreatic cancer cell lines PANC-1 were screened for similar effects. LPA5 absence stimulated cell motility, invasiveness and angiogenesis which evidently showed the anti-cancer role of LPA5. Conversely, these effects were reversed by LPA6 knockdown. LPA5 also reduced the cell motility Mmp-9 activation in fibroblast 3T3 cells [26]and sarcoma cells [27]. Cell motility activity in endothelial cells is majorly regulated by LPA signalling.

The role of LPA in the regulation of matrix metalloproteinase (MMP) is well documented. The ATX-LPA-LPA1 signalling axis has been shown to induce MMP-9 expression in hepatocellular carcinoma (HCC). The expression levels of the Mmp-2 gene in MFHL5-2 cells depleted for Lpar5 were significantly higher than those in control MFHGFP cells [28]. On the other hand, angiogenesis, which is the process of producing new blood vessels in order to promote metastasis, is regulated by various factors, including VEGF. Recently a connection was discovered between LPA signalling and VEGF. In another investigation, endothelial cells were cultured with a conditioned medium from neuroblastoma cells expressing individual LPA receptors, and both LPA1 and LPA3 were shown to stimulate the cell motility of endothelial cells, correlating with the expression levels of VEGF genes [29]. A later study found contrasting results where LPA5 decreased VEGF expression and negatively regulated cell motility [28]. This theory not only explains the protective nature of LPA5 in carcinogenesis but also the role of LPA in angiogenesis signalling establishing a chemotherapeutic target in sarcomas. LPA-induced activation of $\mathrm{G}_{12 / 13}$ and $\mathrm{G}_{q}$ and escalation of cAMP was proven in previous studies. Studies are now denoting a protein GPR92 fifth receptor LPA5 which has similar physiological roles [30]. 
Autotaxin is an ectonucleotide pyrophosphatase/phosphodiesterase (ENPP) family member which produces LPA. Up-regulated in most of the metastatic cancers, autotaxin was also associated with invasiveness and aggressive metastatic potential of cancers and was positively correlated to tumour angiogenesis in colorectal cancer [31]. Furthermore, evidence has identified autotoxins as secretory proteins in human melanoma cells, and the over expression of autotoxins was co-relatable to increased motility and invasiveness [32]. LPA and autotoxin expression differ vastly with regard to in vitro and in vivo settings. Autotaxin was found to be involved in facilitating B16F10 metastasis in C57BL/6 mice [33,34]. However, under in vitro conditions, there was a diminished cell invasion in similar cells under the influence of LPA [35]. Negative effects of LPA5 against cell proliferation and migration have been shown in melanoma and pancreatic cancer cells [27], and the exogenous expression of LPA5 in intestinal epithelial cells MSIE lessened cell proliferation. Such evidence greatly supports the proven role of LPA5 in cancer therapeutics.

Cancer immune editing is a process adapted by cancer cells in order to evade cell death and reside in harsh environments. Some of the mechanisms employed by the tumours in order to escape harm include CD8T cell response. The CD8T activation by tumour antigen is initiated through T cell antigen receptor (TCR) signalling. Previous deleterious effects of LPA on migration, metastasis and therapeutic resistance was underscored in a study by Oda et al., where LPA5 receptor and signalling by this GPCR inhibits CD8 T cell receptor signalling, activation and proliferation [36]. This study not only identified the requirement of LPA5 for negative regulation of TCR-induced calcium mobilisation but also in attenuating antigen-mediated proliferation in vivo.

\section{ER stress: A friend apart from foe?}

Endoplasmic reticulum (ER) strives for homeostasis. When ER function becomes overwhelmed with an excessive accumulation of misfolded proteins within the lumen, ER stress is triggered. ER stress releases coping mechanisms to reduce the damage. The adaptation to a stress environment is achieved by ER stress. If the recovery of cellular adaptation fails, long-standing ER stress triggers programmed cell death or apoptosis. On the other hand, growing evidence suggests a novel pathway which helps cells to survive extreme environmental conditions and escape cell death via upregulation of ER adaptive measures. The million-dollar question here is whether ER stress is a saviour or a killer [37].

Cancer stem cells (CSC) are more resistant towards chemotherapy, consequently sensitising these cells to chemotherapy is a means by which to render them prone to cell death. CSCs treated with salubrinal, a specific inhibitor of eIF2 $\alpha$ phosphatase, followed by conventional chemotherapuetic agents resulted in sensitisation of CSC towards oxaliplatin and 5-FU. A similar pattern was observed during in vivo experiments. Mice treated with salubrinal led to transient UPR activation which increased growth of xenografts derived from colon-CSCs, however combinational treatment with chemotherapeutic agents suppressed the growth of the xenografts, indicating the positive effect of UPR in vitro and in vivo [38].

Conditions such as hypoxia are essential for tumour survival. Several cancers up-regulate GRP78 and XBP1 splicing. In colon cancer, hypoxia induces PERK-dependent phosphorylation of eukaryotic translation initiation factor 2a (eIF2a) and translation of ATF4. UPR is vital for tumour growth under hypoxia. PERK inactivation, due to the generation of mutations in its kinase domain, impairs cell survival under extreme hypoxia, and PERK promotes cancer cell proliferation by limiting oxidative DNA damage through ATF4 [39]. On the other hand, the same ER stress is useful in promoting cell death. Tolfenamic acid promotes ER stress, resulting in activation of the unfolded protein response (UPR)-signalling pathway, of which PERK-mediated phosphorylation of eukaryotic translation initiation factor 2a (eIF2a) induces the repression of cyclin D1 translation. Moreover, the PERK-eIF2a-ATF4 branch of the UPR pathway plays a role in tolfenamic acid-induced apoptosis in colorectal cancer cells, as silencing ATF4 attenuates tolfenamic acid-induced apoptosis.

Plant metabolites have been a promising source of cancer therapeutics for many decades. Esculetin, a coumarin derivative, has been examined on colon cancer cells for its potential anti-cancer activity via ER stress-mediated cell death. Esculetin-induced cell death via the ER stress-mediated 
pathway increased mitochondrial $\mathrm{Ca}^{2+}$ overload and also escalated the level of ER stress response proteins. It was also proven that the cell death was induced by a mechanism of UPR where CHOP is up-regulated and caspase- 12 is cleaved. This CHOP initiation mechanism hinders BCL2 family proteins and activates BAK and BAX, thereby inducing apoptosis [40].

Activation of caspase-3, which is an intrinsic cell death pathway, is another mode of inducing apoptosis. Andrographolide treatment leads to apoptosis in numerous cancer cells. One of the pathways that has been elucidated and which has most potential is ER stress-mediated cancer cell death. Upon treatment, there was a significant increase in IRE1- $\alpha$ and spliced XBP-1 which triggers apoptosis. Surprisingly, the Andrographolide-mediated cell death was also dependent upon ER stress because Andrographolide up-regulated the expression of BAX and also major ER stress markers [41].

Triggering a transcription of heat shock proteins can lead to ER stress-mediated cell death. At the same time, proteasome inhibitors were reported to initiate apoptosis in cancer cells, and functional or mutational changes in some of the ER genes have been associated with malfunctions. XBP1 mutation has been reported in rare myeloma and may be associated with resistance to proteasome inhibitors [42]. Hence it is evident that this inhibition might play a key role in cancer therapeutics. For instance PS-341, a di-peptidyl boronic acid derivative, has shown impressive binding to 26 proteasome and has induced cell death in numerous cancer cell lines. With the ability to target $26 \mathrm{~S}$ proteasome, PS-341 has been logically associated with targeting NF-kB. Traditionally, NF-kB was linked to chemotherapy resistance and PS-341 was tested in order to check its ability in inhibiting NF-kB via chemotherapy-mediated cell death [43]. Surprisingly, it was found that PS-341 could induce topoisomerase-1 inhibitor-mediated apoptosis. Potency of PS-341 was assessed in head and neck SCC (HNSCC) cells [44-46]. PS-341 not only lessened NF-kB but also induced cell death via the ER stress pathway. As part of the involvement of ER stress-modulated cell death in the PS-341 mechanism, caspase-4 played a crucial role. A study by Fribley and Wang described the potential mediatory agents in ER stress-mediated cell death involving PS-341. Their review found that the two major mechanisms involved are BH3-only members of the Bcl2 family interfering with the cytochrome-c release and via induction of BH3-only proteins Bik and Bim[47].

Aspects that need to be emphasised regarding the induction of apoptosis in various tumours include co-expression or loss of genes such asp53 and the time period of ER stress induction. Conceptually, targeting UPR might block ER stress-induced apoptosis and unwittingly promote carcinogenesis. There are few reports of UPR being involved in promoting cancer. Such mechanisms might depend on the intensity and time of the ER stress which we recently proved via dynamics of ER stress [48] and where we have identified the maximum deleterious effect of ER stress at the $6^{\text {th }}$ hour of the dynamics period in vitro. We even employed unconjugated bilirubin to impede ER stressmediated cancer progression in LS174T cells via similar time point [49]. This clearly indicates that the exposure or the ER stress mediation in induction of cell death is critical.

\section{Good guys in the epithelial-mesenchymal transition (EMT) pathway}

Epithelial-mesenchymal transition (EMT) is known to be an important factor associated with cancers' progression, metastasis and treatment resistance[50]. Polarised epithelial cells lose their cellcell adhesion and apical-basal polarity, and obtain the motile, migratory properties of mesenchymal cells [51]. The transition process involves numerous pathological changes. The cells gradually decrease the expression of epithelial cell-cell junction proteins, such as E-cadherin, ZO-1 andcytokeratins, and increase the expression of mesenchymal phenotype, such as N-cadherin, fibronectin and vimentin[52, 53].

There are several signalling pathways driving EMT, including inflammation, transforming growth factor beta (TGF- $\beta$ ), Wnts, NF-kB and Notch pathways [52]. The TGF- $\beta$ pathway, as a primary inducer of EMT, is activated by binding TGF- $\beta$ ligands to their cognate TGF- $\beta$ receptors. With the active TGF- $\beta$ receptors, TGF- $\beta$ signalling complies with Smad 2 and Smad3 to lead to EMT[54]. In addition, TGF- $\beta$ signalling can also stimulate GTPases, PI3K and MAPK pathways to induce EMT 
progression[55]. Other signalling pathways, such as Wnt, Notch, AKT-mTOR and NF-кB pathways, induce EMT by activating EMT transcription factors (EMT-TFs)[52]. There are certain transcription factors described as major regulators of EMT, such as Snail, Twist, $\beta$-catenin, ZEB1 and ZEB2. EMTTFs suppress the expression of epithelial proteins[56]. For instance, Snail inhibits the expression of the key epithelial protein E-cadherin, therefore epithelial cells lose the cell-cell junction formation, leading to mesenchymal transition[57]. The EMT process in normal tissues is managed through a complicated regulation of EMT-TFs, with applied regulatory networks operating at different transcriptional and post-translational levels, such as alternative splicing, non-coding RNAs, epigenetic regulatory mechanisms and protein stability[58]. Studies have shown that the role of EMTTFs in cancer progression is not only to regulate the invasion and dissemination of cancer cells, but also that they play pivotal roles which can become a target of interest for anti-cancer therapy[59].

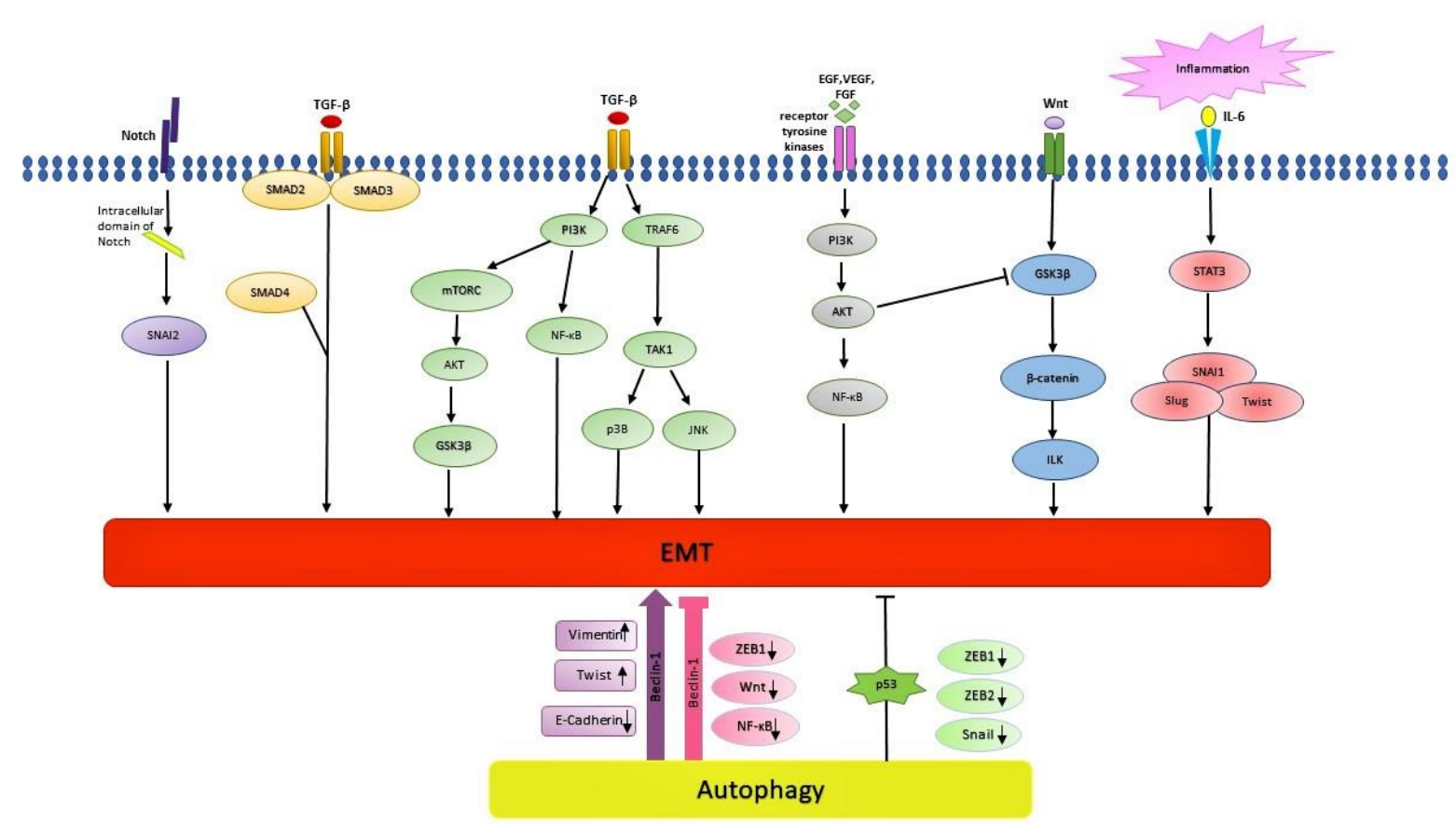

Figure 2. Cross-link between EMT-induced signalling pathways and Autophagy-induced signalling pathways.

EMT-mediated signalling pathway includes Notch, TGF- $\beta$, receptor tyrosine kinases, Wnt, and inflammatory pathways. TGF- $\beta$ signalling complies with SMAD2 and SMAD3 to lead to EMT. TGF$\beta$ signalling also activates PI3K-mTOR-AKT-GSK3b pathway to induce EMT. Wnt signalling drives EMT through GSK3b inhibition and b-catenin stabilization. Wnt, Notch and inflammatory pathways induce EMT by activation of EMT transcription factors, including SNAI1, b-catenin, Twist and Slug. Receptor tyrosine kinases activates PI3K-ATK pathway via activation of growth factors, such as EGF, VEGF and FGF. Autophagy triggers EMT by up-regulating mesenchymal markers including vimentin and Twist and down regulating epithelial marker E-cadherin through Beclin-1 pathway. Beclin-1 pathway can activate autophagy to suppress EMT via down-regulating EMT transcription factors, such as ZEB1 and inhibiting Wnt and NF- $\mathrm{BB}$ pathway. P53 inhibits EMT by decreasing the expression of EMT transcription factors, such as ZEB1/ZEB2, Snail.

Autophagy is another principal biological process involved in the development of cancer, and there is a complex link between autophagy-corresponding and EMT-corresponding signalling pathways (Figure2). Studies have shown that EMT signalling pathways can trigger or inhibit autophagy. As well as being associated with the initiation and suppression of EMT, autophagy also supports EMT in the viability of potentially metastasis of cancer cells [60]. For instance, autophagy deterioration was demonstrated by suppressing autophagy-related genes 5 (ATG5), ATG7 or Beclin1 , resulting in an increase of cell motility and invasiveness with the up-regulation of Snail and Slug, 
two of the major EMT-TFs[61]. On the other hand, autophagy prevents EMT, and the autophagy activation may decrease the gaining of the EMT phenotype in cancer cells. Autophagy is regulated by PI3K/AKT/mTOR, Beclin-1, p53 and JAK/STAT signalling pathways, which have a dramatic impact on the EMT process [59]. EMT-correlated signalling pathways, such as integrin, Wnt, NF- $\kappa \mathrm{B}$, and TGF- $\beta$ signalling pathways, also play an essential role in autophagy[59].

Beclin-1 activates autophagy and accelerates EMT by up-regulating vimentin and Twist expression and decreasing E-cadherin expression [62]. In contrast, Beclin-1 activated autophagy down-regulates MMPs' expression to inhibit EMT and also inhibit EMT via down-regulating ZEB1, Wnt1 and NF- $\kappa$ B. NF- $\kappa B$ activation is associated with aggressiveness and the metastatic potential of carcinomas[63]. The NF- $\kappa B$ pathway promotes EMT by up-regulating related EMT markers, including Snail1, Slug and Twist1 [64], and inhibits autophagy by down-regulating the Beclin-1 pathway [59]. In addition, the study also indicated that Beclin-1 gene knockout may promote EMT and cancerogenesis by activating the Wnt1 and NF- $\kappa B$ pathways resulting in cancer cell metastasis. However, knockdown of Beclin-1 via small interfering RNA (siRNA) suppressed the autophagy activation, consequentially suppressing EMT and the invasiveness of colon cancer cells through cooperating down-regulation of vimentin and Twist and up-regulation of E-cadherin[65]. This result suggests that inhibiting Beclin-1-induced autophagy would be an effective anti-cancer strategy.

P53 is an important suppressor protein of cancer. P53 mediates cancer inhibition by downregulating autophagy-correspond signalling pathways PI3K/AKT/mTOR via interaction with PTEN, which furthers the up-regulation of autophagy[59].P53 can also mediate cancer suppression by regulating EMT inhibition through decreasing the expression of EMT-TFs, including ZEB1, ZEB2 and Snail, via activation of the relevant microRNA of EMT inhibition[66, 67]. Interestingly, mutant p53 can promote EMT and mitochondrial fission that in turn promotes autophagy [68].

Autophagy and EMT both play an important role in the biological processes of induction and development of cancer. Understanding the complicated link between autophagy and EMT is necessary for designing a cancer therapy strategy. Autophagy activation not only supports the cells' survival during the EMT, but also functions as the tumour-suppressive signal, which inhibits the early phase of metastasis and activation of the EMT. Hence, regulating EMT by targeting autophagy is a promising potential strategy for cancer therapy. Currently, translational applications of autophagy activators such as rapamycin, and autophagy inhibitors such as chloroquine and 3methyladenine to regulate the EMT process, have been utilised in anti-cancer therapy $[60,69,70]$.

\section{Conclusion}

Malfunctions in the mutations of GPCR genes are predominantly due to misfolding of mutant receptors in the ER. Understanding the GPCR-mediated mechanisms such as seeding of metastasising tumours by LPA or vice versa and possible role of ER stress in hampering of prosurvival mechanisms in cancers would be extremely important. These hallmarks of events regulate crucial mechanisms such as EMT which is the most pivotal step in metastasis. Hence, a proper elucidation of these candidates would help in identifying potent molecular targets for regulation or modulation of tumour progression in numerous cancers.

Author Contributions: RG designed and conceptualized the review. WL helped in designing and drafting areas like EMT. WL also aided in designing figure2. RE helped in checking and finalizing the review.

\section{Funding: NA}

\section{Acknowledgments: NA}

Conflicts of Interest: The authors declare no conflict of interest. The funders had no role in the design of the study; in the collection, analyses, or interpretation of data; in the writing of the manuscript, and in the decision to publish the results.

\section{References:}


1. Kastner S, Voss T, Keuerleber S, Glöckel C, Freissmuth M, Sommergruber W. Expression of G Protein-Coupled Receptor 19 in Human Lung Cancer Cells Is Triggered by Entry Into S-phase and Supports G(2)-M Cell-Cycle Progression. Mol Cancer Res. 2012;10:1343-58.

2. Yu S, Sun L, Jiao Y, Lee LTO. The Role of G Protein-coupled Receptor Kinases in Cancer. Int J Biol Sci. 2018;14:189-203.

3. Wu V, Yeerna H, Nohata N, Chiou J, Harismendy O, Raimondi F, et al. Illuminating the Onco-GPCRome: Novel G protein-coupled receptor-driven oncocrine networks and targets for cancer immunotherapy. J Biol Chem. 2019;294:11062-86.

4. Maeyashiki C, Melhem H, Hering L, Baebler K, Cosin-Roger J, Schefer F, et al. Activation of pH-Sensing Receptor OGR1 (GPR68) Induces ER Stress Via the IRE1 $\alpha /$ JNK Pathway in an Intestinal Epithelial Cell Model. Sci Rep. 2020;10:1438.

5. Styer KL, Singh V, Macosko E, Steele SE, Bargmann CI, Aballay A. Innate immunity in Caenorhabditis elegans is regulated by neurons expressing NPR-1/GPCR. Science. 2008;322:460-4.

6. Sternberg EM. Neural regulation of innate immunity: a coordinated nonspecific host response to pathogens. Nat Rev Immunol. 2006;6:318-28.

7. Coly PM, Perzo N, Le Joncour V, Lecointre C, Schouft MT, Desrues L, et al. Chemotactic G protein-coupled receptors control cell migration by repressing autophagosome biogenesis. Autophagy. 2016;12:2344-62.

8. Thomson S, Petti F, Sujka-Kwok I, Epstein D, Haley JD. Kinase Switching in Mesenchymal-Like Non-Small Cell Lung Cancer Lines Contributes to EGFR Inhibitor Resistance Through Pathway Redundancy. Clin Exp Metastasis. 2008;25:843-54.

9. Mahmood MQ, Ward C, Muller HK, Sohal SS, Walters EH. Epithelial mesenchymal transition (EMT) and non-small cell lung cancer (NSCLC): a mutual association with airway disease. Med Oncol. 2017;34:45.

10. Filardo EJ, Quinn JA, Bland KI, Jr ARF. Estrogen-induced activation of Erk-1 and Erk-2 requires the G proteincoupled receptor homolog, GPR30, and occurs via trans-activation of the epidermal growth factor receptor through release of HB-EGF. Mol Endocrinol. 2000;14:1649-60.

11. Hart S, Fischer OM, Prenzel N, Zwick-Wallasch E, Schneider M, Hennighausen L, et al. GPCR-induced migration of breast carcinoma cells depends on both EGFR signal transactivation and EGFR-independent pathways. Biol Chem. 2005;386:845-55.

12. So Hee Dho 12 K-PL, Dongjun Jeong 3, Chang-Jin Kim 3, Kyung-Sook Chung 4, Ji Young Kim 1, Bum-Chan Park 1, Sung Sup Park 1, Seon-Young Kim 4 5, Ki-Sun Kwon. GPR171 expression enhances proliferation and metastasis of lung cancer cells. Oncotarget. 2016;7:7856-65.

13. O'Hayre M, Degese MS, Gutkind JS. Novel insights into G protein and G protein-coupled receptor signaling in cancer Curr Opin Cell Biol. 2014;27:126-35.

14. Lutz S, Shankaranarayanan A, Coco C, Ridilla M, Nance MR, Vettel C, et al. Structure of Galphaq-p63RhoGEFRhoA complex reveals a pathway for the activation of RhoA by GPCRs. Science. 2007;318:1923-7.

15. Momotani K, Artamonov MV, Utepbergenov D, Derewenda U, Derewenda ZS, Somlyo AV. p63RhoGEF Couples $\mathrm{G} \alpha(\mathrm{q} / 11)$-mediated Signaling to Ca2+ Sensitization of Vascular Smooth Muscle Contractility. Circ Res. 2011;109:993-1002.

16. Jones PG, Nawoschik SP, Sreekumar K, Uveges AJ, Tseng E, Zhang L, et al. Tissue Distribution and Functional Analyses of the Constitutively Active Orphan G Protein Coupled Receptors, GPR26 and GPR78. Biochim BIophys Acta. 2007;1770:890-901.

17. Dong D-D, Zhou H, Li G. GPR78 promotes lung cancer cell migration and metastasis by activation of Gaq-Rho GTPase pathway. BMB Rep. 2016;49:623-8.

18. Hall A. Rho Family GTPases. Biochem Soc Trans. 2012;40:1378-82.

19. Gulhati P, Bowen KA, Liu J, Stevens PD, Rychahou PG, Chen M, et al. mTORC1 and mTORC2 regulate EMT, motility and metastasis of colorectal cancer via RhoA and Rac1 signaling pathways Cancer Research. 2011;71:324656.

20. Zhong Q, Zhou B, Ann DK, Minoo P, Liu Y, Banfalvi A, et al. Role of endoplasmic reticulum stress in epithelialmesenchymal transition of alveolar epithelial cells: effects of misfolded surfactant protein. Am J Respir Cell Mol Biol. 2011;45:498-509.

21. Delbrel E, Uzunhan Y, Soumare A, Gille T, Marchant D, Planès C, et al. ER Stress is Involved in Epithelial-ToMesenchymal Transition of Alveolar Epithelial Cells Exposed to a Hypoxic Microenvironment. Int J Mol Sci. 2019;20. 
22. Zhou S, Yang J, Wang M, Zheng D, Liu Y. Endoplasmic reticulum stress regulates epithelial-mesenchymal transition in human lens epithelial cells. Mol Med Rep. 2020;21:173-80.

23. Liu D, Zhu H, Gong L, Pu S, Wu Y, Zhang W, et al. Histone Deacetylases Promote ER Stress Induced Epithelial Mesenchymal Transition in Human Lung Epithelial Cells. Cell Physiol Biochem. 2018;46:1821-34.

24. Minami K, Ueda N, Ishimoto K, Tsujiuchi T. LPA(5)-mediated signaling induced by endothelial cells and anticancer drug regulates cellular functions of osteosarcoma cells. Exp Cell Res. 2020;388:111813.

25. Jongsma M, Matas-Rico E, Rzadkowski A, Jalink K, Moolenaar WH. LPA is a chemorepellent for B16 melanoma cells: action through the cAMP-elevating LPA5 receptor. PLoS One. 2011;6:e29260.

26. Dong Y, Hirane M, Araki M, Fukushima N, Tsujiuchi T. Lysophosphatidic acid receptor-5 negatively regulates cellular responses in mouse fibroblast 3T3 cells. Biochem Biophys Res Commun. 2014;446:585-9.

27. Ishii S, Hirane M, Fukushima K, Tomimatsu A, Fukushima N, Tsujiuchi T. Diverse effects of LPA4, LPA5 and LPA6 on the activation of tumor progression in pancreatic cancer cells. Biochem Biophys Res Commun. 2015;461:59-64.

28. Araki M, Kitayoshi M, Dong Y, Hirane M, Ozaki S, Mori S, et al. Inhibitory effects of lysophosphatidic acid receptor5 on cellular functions of sarcoma cells. Growth Factors. 2014;32:117-22.

29. Kitayoshi M, Kato K, Tanabe E, Yoshikawa K, Fukui R, Fukushima N, et al. Enhancement of endothelial cell migration by constitutively active LPA(1)-expressing tumor cells. Biochem Biophys Res Commun. 2012;422:339-43.

30. Lee CW, Rivera R, Gardell S, Dubin AE, Chun J. GPR92 as a new G12/13- and Gq-coupled lysophosphatidic acid receptor that increases cAMP, LPA5. J Biol Chem. 2006;281:23589-97.

31. Yun CC. Lysophosphatidic Acid and Autotaxin-associated Effects on the Initiation and Progression of Colorectal Cancer. Cancers (Basel). 2019;11.

32. Lee SC, Fujiwara Y, Liu J, Yue J, Shimizu Y, Norman DD, et al. Autotaxin and LPA1 and LPA5 receptors exert disparate functions in tumor cells versus the host tissue microenvironment in melanoma invasion and metastasis. Mol Cancer Res. 2015;13:174-85.

33. Gotoh M, Fujiwara Y, Yue J, Liu J, Lee S, Fells J, et al. Controlling cancer through the autotaxin-lysophosphatidic acid receptor axis. Biochem Soc Trans. 2012;40:31-6.

34. Gupte R, Patil R, Liu J, Wang Y, Lee SC, Fujiwara Y, et al. Benzyl and naphthalene methylphosphonic acid inhibitors of autotaxin with anti-invasive and anti-metastatic activity. ChemMedChem. 2011;6:922-35.

35. Lee SC, Fujiwara Y, Tigyi GJ. Uncovering unique roles of LPA receptors in the tumor microenvironment. Receptors Clin Investig. 2015;2.

36. Oda SK, Strauch P, Fujiwara Y, Al-Shami A, Oravecz T, Tigyi G, et al. Lysophosphatidic acid inhibits CD8 T cell activation and control of tumor progression. Cancer Immunol Res. 2013;1:245-55.

37. Wang WA, Groenendyk J, Michalak M. Endoplasmic reticulum stress associated responses in cancer. Biochim Biophys Acta. 2014;1843:2143-9.

38. Wielenga MCB, Colak S, Heijmans J, van Lidth de Jeude JF, Rodermond HM, Paton JC, et al. ER-Stress-Induced Differentiation Sensitizes Colon Cancer Stem Cells to Chemotherapy. Cell Rep. 2015;13:489-94.

39. Vandewynckel YP, Laukens D, Geerts A, Bogaerts E, Paridaens A, Verhelst X, et al. The paradox of the unfolded protein response in cancer. Anticancer Res. 2013;33:4683-94.

40. Kim AD, Madduma Hewage SR, Piao MJ, Kang KA, Cho SJ, Hyun JW. Esculetin induces apoptosis in human colon cancer cells by inducing endoplasmic reticulum stress. Cell Biochem Funct. 2015;33:487-94.

41. Banerjee A, Ahmed H, Yang P, Czinn SJ, Blanchard TG. Endoplasmic reticulum stress and IRE-1 signaling cause apoptosis in colon cancer cells in response to andrographolide treatment. Oncotarget. 2016;7:41432-44.

42. Hong SY, Hagen T. Multiple myeloma Leu167Ile (c.499C>A) mutation prevents XBP1 mRNA splicing. Br J Haematol. 2013;161:898-901.

43. Cusack JC, Jr., Liu R, Houston M, Abendroth K, Elliott PJ, Adams J, et al. Enhanced chemosensitivity to CPT-11 with proteasome inhibitor PS-341: implications for systemic nuclear factor-kappaB inhibition. Cancer Res. 2001;61:3535-40.

44. Denlinger CE, Rundall BK, Jones DR. Proteasome inhibition sensitizes non-small cell lung cancer to histone deacetylase inhibitor-induced apoptosis through the generation of reactive oxygen species. J Thorac Cardiovasc Surg. 2004; $128: 740-8$.

45. Yeung BH, Huang DC, Sinicrope FA. PS-341 (bortezomib) induces lysosomal cathepsin B release and a caspase-2dependent mitochondrial permeabilization and apoptosis in human pancreatic cancer cells. J Biol Chem. 2006;281:11923-32. 
46. Pérez-Galán P, Roué G, Villamor N, Montserrat E, Campo E, Colomer D. The proteasome inhibitor bortezomib induces apoptosis in mantle-cell lymphoma through generation of ROS and Noxa activation independent of p53 status. Blood. 2006;107:257-64.

47. Fribley A, Wang CY. Proteasome inhibitor induces apoptosis through induction of endoplasmic reticulum stress. Cancer Biol Ther. 2006;5:745-8.

48. Gundamaraju R, Vemuri R, Chong WC, Myers S, Norouzi S, Shastri MD, et al. Interplay between Endoplasmic Reticular Stress and Survivin in Colonic Epithelial Cells. Cells. 2018;7.

49. Gundamaraju R, Vemuri R, Chong WC, Bulmer AC, Eri R. Bilirubin Attenuates ER Stress-Mediated Inflammation, Escalates Apoptosis and Reduces Proliferation in the LS174T Colonic Epithelial Cell Line. Int J Med Sci. 2019;16:135-44.

50. Pastushenko I, Blanpain C. EMT Transition States during Tumor Progression and Metastasis. Trends Cell Biol. 2019;29:212-26.

51. Goossens S, Vandamme N, Van Vlierberghe P, Berx G. EMT transcription factors in cancer development reevaluated: Beyond EMT and MET. Biochim Biophys Acta Rev Cancer. 2017;1868:584-91.

52. Du B, Shim JS. Targeting Epithelial-Mesenchymal Transition (EMT) to Overcome Drug Resistance in Cancer. Molecules. 2016;21.

53. Lamouille S, Xu J, Derynck R. Molecular mechanisms of epithelial-mesenchymal transition. Nat Rev Mol Cell Biol. 2014;15:178-96.

54. Kaimori A, Potter J, Kaimori JY, Wang C, Mezey E, Koteish A. Transforming growth factor-beta1 induces an epithelial-to-mesenchymal transition state in mouse hepatocytes in vitro. J Biol Chem. 2007;282:22089-101.

55. Moustakas A, Heldin CH. Non-Smad TGF-beta signals. J Cell Sci. 2005;118:3573-84.

56. Caramel J, Ligier M, Puisieux A. Pleiotropic Roles for ZEB1 in Cancer. Cancer Res. 2018;78:30-5.

57. Wang Y, Shi J, Chai K, Ying X, Zhou BP. The Role of Snail in EMT and Tumorigenesis. Curr Cancer Drug Targets. 2013;13:963-72.

58. Santamaria PG, Moreno-Bueno G, Portillo F, Cano A. EMT: Present and future in clinical oncology. Mol Oncol. 2017;11:718-38.

59. Chen HT, Liu H, Mao MJ, Tan Y, Mo XQ, Meng XJ, et al. Crosstalk between autophagy and epithelial-mesenchymal transition and its application in cancer therapy. Mol Cancer. 2019;18:101.

60. Singla M, Bhattacharyya S. Autophagy as a potential therapeutic target during epithelial to mesenchymal transition in renal cell carcinoma: An in vitro study. Biomed Pharmacother. 2017;94:332-40.

61. Catalano M, D'Alessandro G, Lepore F, Corazzari M, Caldarola S, Valacca C, et al. Autophagy induction impairs migration and invasion by reversing EMT in glioblastoma cells. Mol Oncol. 2015;9:1612-25.

62. Yang M, Zhao H, Guo L, Zhang Q, Zhao L, Bai S, et al. Autophagy-based survival prognosis in human colorectal carcinoma. Oncotarget. 2015;6:7084-103.

63. Huber MA, Kraut N, Beug H. Molecular requirements for epithelial-mesenchymal transition during tumor progression. Curr Opin Cell Biol. 2005;17:548-58.

64. Pires BR, Mencalha AL, Ferreira GM, de Souza WF, Morgado-Díaz JA, Maia AM, et al. NF-kappaB Is Involved in the Regulation of EMT Genes in Breast Cancer Cells. PLoS One. 2017;12:e0169622.

65. Shen H, Yin L, Deng G, Guo C, Han Y, Li Y, et al. Knockdown of Beclin-1 impairs epithelial-mesenchymal transition of colon cancer cells. J Cell Biochem. 2018;119:7022-31.

66. Chang CJ, Chao CH, Xia W, Yang JY, Xiong Y, Li CW, et al. p53 regulates epithelial-mesenchymal transition and stem cell properties through modulating miRNAs. Nat Cell Biol. 2011;13:317-23.

67. Kim T, Veronese A, Pichiorri F, Lee TJ, Jeon YJ, Volinia S, et al. p53 regulates epithelial-mesenchymal transition through microRNAs targeting ZEB1 and ZEB2. J Exp Med. 2011;208:875-83.

68. Zhang X, Cheng Q, Yin H, Yang G. Regulation of autophagy and EMT by the interplay between p53 and RAS during cancer progression (Review). Int J Oncol. 2017;51:18-24.

69. Tong H, Yin H, Hossain MA, Wang Y, Wu F, Dong X, et al. Starvation-induced autophagy promotes the invasion and migration of human bladder cancer cells via TGF- $\beta 1 / \mathrm{Smad} 3$-mediated epithelial-mesenchymal transition activation. J Cell Biochem. 2019;120:5118-27.

70. Zong H, Yin B, Zhou H, Cai D, Ma B, Xiang Y. Inhibition of mTOR pathway attenuates migration and invasion of gallbladder cancer via EMT inhibition. Mol Biol Rep. 2014;41:4507-12. 